J. Lake Sci. (湖泊科学) , 2016, 28(5): 1148-1152

DOI 10. 18307/2016. 0526

(c) 2016 by Journal of Lake Sciences

\title{
浮游植物叶绿素 $\mathrm{a}$ 的微波法研究及其与反复冻融法的比较
}

\author{
林罗敏 ${ }^{1}$, 唐鹊辉 ${ }^{1}$, 彭 亮 $^{1}$, 韦桂峰 $^{1 * *}$, 林叔忠 $^{2}$, 李湘姣 $^{2}$, 杨浩文 ${ }^{2}$ \\ (1:暨南大学生态学系,热带亚热带水生态工程教育部工程研究中心,广州 510632) \\ (2:广东省水文局,广州 510150)
}

\begin{abstract}
摘 要: 为缩短样品处理时间和提高测定准确度, 本文设计和优化了微波处理辅助提取浮游植物叶绿素 $\mathrm{a}$ 的方法, 并比 较了反复冻融法和微波法对浮游植物叶绿素 $\mathrm{a}$ 的提取效率. 结果表明: (1) 微波法提取叶绿素 $\mathrm{a}$ 的最优处理条件为: 高火 (额定输出功率 $800 \mathrm{~W}$ ) 处理 $60 \mathrm{~s}$ 左右. 过滤水量为: 贫营养型水体为 $1000 \mathrm{ml}$ 以上, 中、富营养水体为 $100 \sim 500 \mathrm{ml}$. (2) 反 复冻融法在测定贫营养型水体时更稳定, 而微波法对中、富营养水样提取率显著高于冻融法, 可提高 $7 \% \sim 12 \%$, 对具胶被 及硅质外壳的藻类提取效率极显著高于冻融法,测定结果的相对偏差更小, 且提取时间较冻融法缩短一半以上,适用于 富营养化水体的应急监测.
\end{abstract}

关键词: 微波法;反复冻融法;提取;浮游植物;叶绿素 a

\section{Microwave-assisted method for extracting chlorophyll-a in phytoplankton and its compari- son with freezing-thawing extraction method}

LIN Luomin ${ }^{1}$, TANG Quehui ${ }^{1}$, PENG Liang ${ }^{1}$, WEI Guifeng ${ }^{1 * *}$, LIN Shuzhong $^{2}$, LI Xiangjiao ${ }^{2}$ \& YANG Haowen $^{2}$

(1: Engineering Research Center of Tropical and Subtropical Aquatic Ecological Engineering Ministry of Education, Department of Ecology, Jinan University, Guangzhou 510632, P.R.China)

(2: Hydrological Bureau of Guangdong Province, Guangzhou 510150, P.R.China)

\begin{abstract}
In order to save time of sample processing and to improve the determining accuracy, a microwave-assisted extraction method was designed and optimized to extract chlorophyll-a in phytoplankton, and was compared to the freezing-thawing method for extraction efficiency of chlorophyll-a. The results showed that microwave-assisted extraction method was optimal in $60 \mathrm{~s}$ treatment with output power of $800 \mathrm{~W}$, and the appropriate water volume for filtration was about 100-500 ml for samples collected in mesoeutrophic waterbodies and $1000 \mathrm{ml}$ for those from oligotrophic waterbodies. The chlorophyll extraction rate of microwave-assisted extraction method was significantly higher than that of freezing-thawing method in meso-eutrophic waterbodies, which can increased by $7 \%-12 \%$, while freezing-thawing method was more stable in measuring chlorophyll-a of oligotrophic waterbodies. For those algae with a glue shell or siliceous shell, microwave-assisted extraction method had a higher efficiency for chlorophyll-a extraction and a lower relative standard deviation than the freeze-thaw method. The microwave-assisted extraction method needs only one half operation time of the freezing-thawing extraction method, so that it is more suitable for emergency monitoring of eutrophic waterbodies, while the freezing-thawing extraction method is more suitable for monitoring oligotrophic waterbodies.

Keywords: Microwave-assisted extraction method; freezing-thawing method; extraction; phytoplankton; chlorophyll-a
\end{abstract}

叶绿素 $\mathrm{a}$ 是表征藻类现存量的一个重要指标 ${ }^{[1]}$, 可在一定程度上反映水体初级生产力和浮游植物生物 量 ${ }^{[2]}$, 是水体富营养化评价的重要指标和水华预警的重要依据 ${ }^{\left[{ }^{[3]}\right.}$. 因此, 叶绿素 a 浓度的准确测定是水体富 营养化研究和水质评价的重要基础 ${ }^{[1]}$. 在叶绿素 $\mathrm{a}$ 的测定过程中, 较高的提取率是保证测定结果准确性和

* 广东省水利科技创新项目“广东省河流水生态健康评价指标体系及评价方法研究” (2014-01) 资助.2015-10-23 收稿;2015 - 12 - 16 收修改稿. 林罗敏(1990 ), 女, 硕士研究生; E-mail: linluomin@126.com.

** 通信作者;E-mail:tweigf@jnu.edu.cn. 
稳定性的前提. 目前常用的提取方法是利用反复冻融细胞破碎藻类细胞, 再用丙酮或乙醇等有机溶剂浸提 细胞溶出物中的叶绿素 ${ }^{[4-7]}$, 该方法冻融操作简单, 与有毒溶剂接触时间短 ${ }^{[7-8]}$, 但耗时相对较长, 且可能对 具胶被或硅质壳藻细胞的破碎能力较弱.

自 1986 年开始利用微波技术从羽扇豆中提取鹰爪豆生物碱以来 ${ }^{[9]}$, 微波辅助技术 ( microwave-assisted extraction, MAE) 在色素的提取工艺中被广泛应用 ${ }^{[10-12]}$. 微波法通过升温汽化胞内液体,使细胞膨胀破裂, 内 容物自由流出, 因此萃取率较高 ${ }^{[13]}$. 但此方法对浮游植物细胞尤其是具胶被或硅质壳藻细胞是否具有较强 的破碎能力和较高的叶绿素提取效果还有待验证. 为缩短样品处理时间和提高测定的准确度, 本文设计并 优化了微波法提取浮游植物叶绿素 $\mathrm{a}$ 的处理条件, 比较了优化后的微波法和反复冻融法 (Freezing-thawing, FT) 对叶绿素 a 的提取率及两种方法的稳定性,提出适合不同营养水平水体叶绿素 $\mathrm{a}$ 的测定方法.

\section{1 材料与方法}

\section{1 实验材料}

纯藻液样品: 实验室培养的铜绿微囊藻 (Microcystis aeruginosa)、蛋白核小球藻 (Chlorella pyrenoidosa) 和 颗粒直链藻 (Melosira granulata).

野外样品: 湖泊 (暨南大学南湖 S1 、明湖 S2)、河流( 流溪河石海桥 S3、珠江猎德大桥 S4)、水库 ( 流溪河 水库 S5、南屏水库 S6), 其中 S1、S3、S5 叶绿素浓度较低,S2、S4、S6 叶绿素浓度较高.

\section{2 实验方法}

实验内容包括微波处理条件优化及其与反复冻融法提取叶绿素效果比较两部分, 具体实验设计见表 1. 1.2.1 微波法操作步骤及实验条件优化 用醋酸纤维滤膜 $(0.45 \mu \mathrm{m})$ 过滤一定量的水样, 再将滤膜折叠为 $1 / 4$ 后, 置于对折的定性滤纸中遮光并吸去滤膜背面水分. 将滤膜连同定性滤纸和装有小半杯水的烧杯 (防止微 波炉空烧) 放人微波炉 (型号:P80D23N2L-A9(R0), 额定输出功率 $800 \mathrm{~W}$ ) 中, 在高火 (额定输出功率 100\% 输出, 即 $800 \mathrm{~W}$ 功率输出 $\left.{ }^{[14]}\right)$ 条件下进行微波破壁处理.

破壁处理后将滤膜取出放人盛有 $10 \mathrm{ml} 90 \%$ 丙酮溶液的离心管中, 用快速混匀器/手摇震荡 $1 \mathrm{~min}$ 后放 于 $4^{\circ} \mathrm{C}$ 冰箱浸提 $8 \mathrm{~h}$ 以上,再经过 7000 转 $/ \min$ 离心 $15 \mathrm{~min}$ 后, 用 Hitachi U-3900 紫外分光光度计于 630 、 $645 、 663$ 和 $750 \mathrm{~nm}$ 波长测量吸光值后计算得出叶绿素 a 浓度 ${ }^{[15]}$.

根据叶绿素 $\mathrm{a}$ 的测定结果, 确定微波法中最优的微波处理时长和过滤水量.

1.2.2 比较微波法和反复冻融法提取效果 分别用微波法和反复冻融法 $\left(-20^{\circ} \mathrm{C}\right.$ 冷冻 $20 \mathrm{~min}$, 取出室温融解 $5 \sim$ $10 \mathrm{~min}$, 反复冻融 5 次, $90 \%$ 丙䣳 $4^{\circ} \mathrm{C}$ 浸提 $20 \mathrm{~h}$ ) ${ }^{[8]}$ 提取纯藻液 (铜绿微囊藻、蛋白核小球藻和颗粒直链藻) 以 及野外水样 $(\mathrm{S} 1 、 \mathrm{~S} 2 、 \mathrm{~S} 3 、 \mathrm{~S} 4 、 \mathrm{~S} 5$ 和 S6) 的叶绿素 a 浓度. 微波法和反复冻融法样品统一批次、浓度及过滤水量 等条件,保证结果的可比性;所有实验设置 5 组平行,保证重复性.

表 1 实验设计*

Tab.1 Experimental design

\begin{tabular}{|c|c|c|c|c|c|}
\hline \multirow[b]{3}{*}{ 水样来源 } & \multicolumn{3}{|c|}{ 微波法处理条件优化 } & \multicolumn{2}{|c|}{ 微波法与反复冻融法比较 } \\
\hline & \multicolumn{2}{|c|}{$\begin{array}{l}\text { 不同微波处理时间 } \\
\text { 对提取效果的影响 }\end{array}$} & \multirow{2}{*}{$\begin{array}{c}\text { 过滤水量对微波法 } \\
\text { 提取效果的影响 } \\
\text { S2 }\end{array}$} & \multirow{2}{*}{$\begin{array}{l}\text { 纯培养藻实验 } \\
\text { Mic.、Chl. Mel. }\end{array}$} & \multirow{2}{*}{$\begin{array}{c}\text { 野外水样实验 } \\
\mathrm{S} 1 、 \mathrm{~S} 2 、 \mathrm{~S} 3 、 \mathrm{~S} 4 、 \mathrm{~S} 5 、 \mathrm{~S} 6\end{array}$} \\
\hline & $\mathrm{S} 1$ & Mic. 、Chl. & & & \\
\hline 稀释倍数 & - & $\begin{array}{c}10 \mathrm{x}, 5 \mathrm{x}, 2.5 \mathrm{x}, \mathrm{x} ; \\
\mathrm{x}<5 \mu \mathrm{g} / \mathrm{L}\end{array}$ & - & $\begin{array}{l}\text { 稀释至中/ } \\
\text { 富营养水平 }\end{array}$ & - \\
\hline 过滤水量/ml & 100 & 100 & $50 、 100 、 500 、 1000$ & $100 \sim 500$ & $100 \sim 500$ \\
\hline 微波处理时间/s & $30 、 60 、 90 、 120 、 150$ & $60 、 80 、 100 、 120$ & 60 & 60 & 60 \\
\hline
\end{tabular}

$* M i c$ : 铜绿微囊藻; Chl. : 蛋白核小球藻; $M e l$. : 颗粒直链藻.

\section{3 数据分析与绘图}

利用 SPSS 20.0 进行单因素方差分析; 用 Origin 8.0 软件完成绘图. 


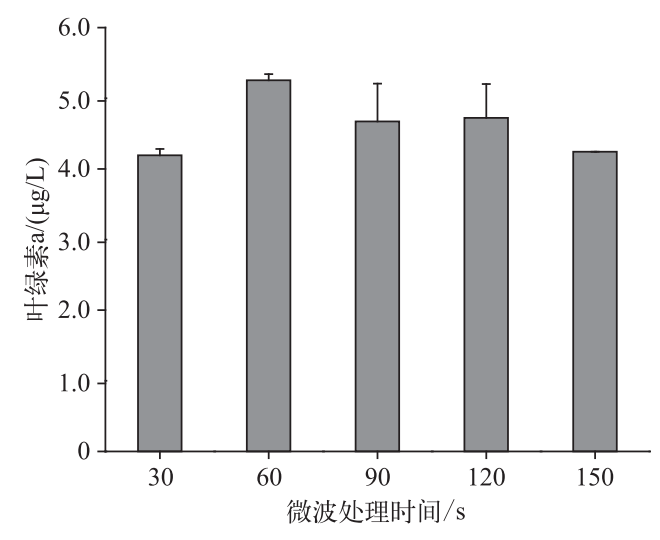

图 1 不同微波处理时间对野外水样 $(\mathrm{S} 1)$ 叶绿素 a 浓度测定结果的影响

\section{2 结果与讨论}

\section{1 微波法的处理条件篮选}

2.1.1 微波处理时间对叶绿素提取效果的影响微 波法主要通过微波破壁产生裂缝和空隙 ${ }^{[1-12]}$, 有助 于有机溶剂丙酮有效地溶出叶绿素. 微波处理时长 直接影响提取效果. 对于野外水体 (S1), 微波处理 $60 \mathrm{~s}$ 后的叶绿素 a 浓度的测定结果显著高于处理 $30 \mathrm{~s}$ 后的测定结果 $(P<0.05)$, 且相对标准偏差较小 (图 1). 但随着处理时间的增加, 叶绿素 a 浓度的测 定结果并未显著升高, 当处理时间达 $150 \mathrm{~s}$ 时, 微波 可能破坏了藻细胞体内的叶绿素 $\mathrm{a}$ 结构 ${ }^{[15]}$, 导致测 定的叶绿素 a 浓度显著下降 $(P<0.05)$. 对于室内培 养的不同浓度的微囊藻藻液和小球藻藻液, 微波处

Fig.1 Effect of microwave treatment time on determination 理时间超过 $60 \mathrm{~s}$ 时, 叶绿素 a 浓度的测定结果无显 of chlorophyll-a concentration from field water samples

著差异 $(P>0.05)$ ( 图 2). 因此, 最适的微波破壁处 理时长为 $60 \mathrm{~s}$ 左右.
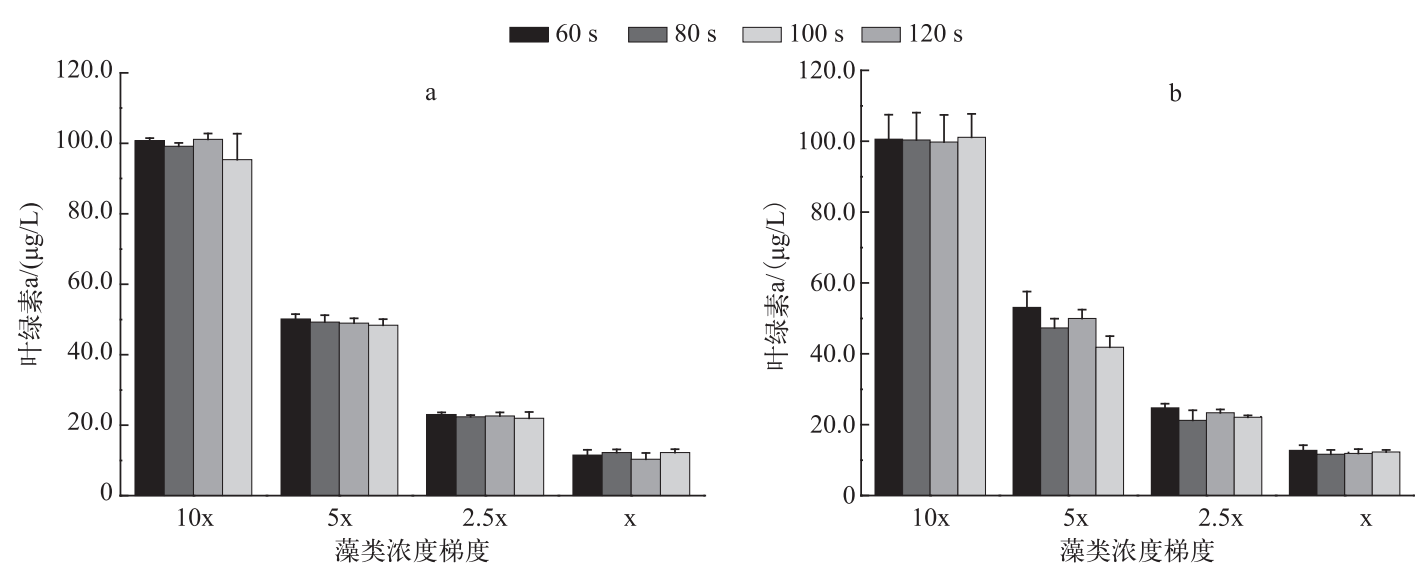

图 2 不同微波处理时间对室内培养藻液叶绿素 $\mathrm{a}$ 浓度的影响 ( $\mathrm{a}$ : 铜绿微囊藻; $\mathrm{b}$ :蛋白核小球藻)

Fig. 2 Effect of microwave irradiation time on determination of chlorophyll-a concentration from cultured samples ( a: Microcystis aeruginosa; b: Chlorella pyrenoidosa)

2.1.2 过滤水量对微波法提取效果的影响 过滤水量为 $50 \mathrm{ml}$ 时, $\mathrm{S} 2$ 水样的叶绿素 a 提取效果较差, 可能是 由于此时滤膜上叶绿素过少, 浓度低于检测限, 导致测量值偏低 (图 3). 当过滤水量增加到 $100 、 500 \mathrm{ml}$ 时, 提取效果显著提高 $(P<0.05)$, 但当过滤水量为 $1000 \mathrm{ml}$ 时, 叶绿素 a 浓度测定值略降低 (图 3), 这可能与过 滤时间较长, 叶绿素部分分解有关 ${ }^{[16]}$, 也可能与泥沙等杂质增多, 干扰测定结果的准确性有关. 因此, 以叶 绿素 a 浓度为 $15 \mu \mathrm{g} / \mathrm{L}$ 左右的富营养水样为例, 微波法提取叶绿素 $\mathrm{a}$ 的最适过滤水量为 $100 \sim 500 \mathrm{ml}$. 随着叶 绿素 a 浓度的升高或降低, 应相应调整过滤水量: 贫营养型水体为 $1000 \mathrm{ml}$; 中、富营养水体为 $100 \sim 500 \mathrm{ml}$.

\section{2 微波法和反复冻融法对叶绿素 a 提取效果的比较}

对于室内培养的微囊藻藻液,微波法和反复冻融法对叶绿素 $\mathrm{a}$ 的提取效果无显著差异 (图 4a), 这可能 与微囊藻细胞体积小、细胞壁薄 ${ }^{88]}$ 有关; 对于室内培养的小球藻和直链藻藻液, 微波法提取效果显著高于反 复冻融法 $(P<0.05)$, 这可能是因为微波辐射均匀, 选择性强 ${ }^{[13]}$, 对具细胞个体胶被的小球藻和具硅质外壳 的直链藻细胞壁的破碎效果较好. 而反复冻融法通过冷冻使细胞内形成冰粒和细胞液浓度增高引起溶 
胀 $^{[17]}$, 依赖多次反复冷冻及室温解冻, 受环境因素影响 较大, 破壁效果较差.

用微波法和反复冻融法分别测定以蓝、绿藻占优势 的湖泊和水库水样以及以硅藻占优势的河流水样中的 叶绿素 a 浓度. 当叶绿素 a 浓度较低时 $(\mathrm{S} 1 、 \mathrm{~S} 3$ 和 S5), 两种方法的测定结果无显著差异; 当叶绿素浓度较高 $(\mathrm{S} 2 、 \mathrm{~S} 4$ 和 S6) 时, 微波法的测定结果显著高于反复冻 融法 $(P<0.05)$, 且相对标准偏差较小, 此时微波法的提 取效果更好( 图 4b) 。

\section{3 结论}

1 ) 微波法提取叶绿素 $\mathrm{a}$ 的最优处理条件为: 高火 (额定输出功率 $800 \mathrm{~W}$ ) $60 \mathrm{~s}$ 左右. 最佳过滤水量为: 贫 营养型水体为 $1000 \mathrm{ml}$; 中、富营养水体为 $100 \sim 500 \mathrm{ml}$.

2 ) 微波法提取对具有胶被和硅质外壳的藻类提取

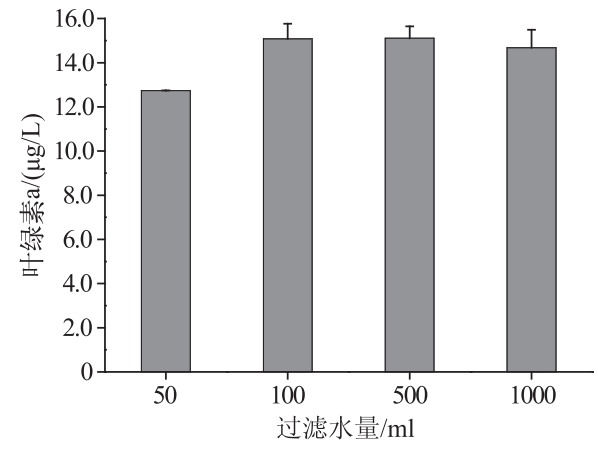

图 3 微波法对不同体积野外水样(S2) 叶绿素 a 浓度的测定结果

Fig.3 The determination of chlorophyll-a concentration in field samples with different volumes by MAE 效率优于冻融法, 且误差更小.
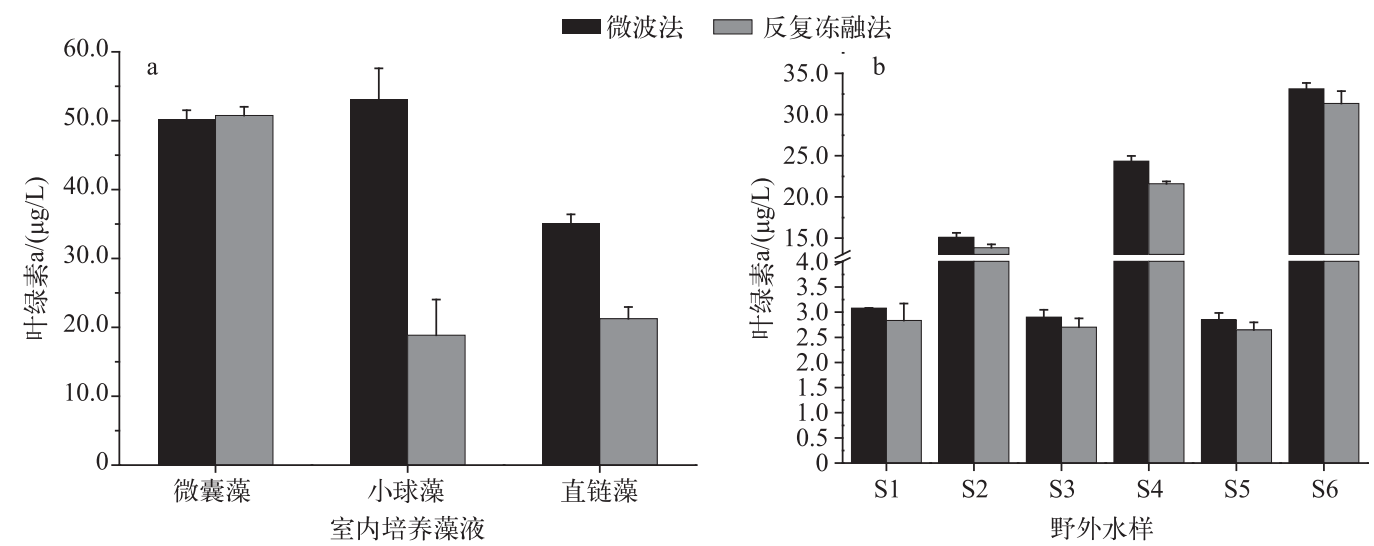

图 4 微波法和反复冻融法对室内培养藻液 (a) 和野外水样 (b) 叶绿素 a 浓度测定结果的比较

Fig.4 Comparison of determination of chlorophyll-a concentration in cultured samples(a) and in field samples(b) by MAE and FT

3 ) 两种方法在操作上均较简单易行. 反复冻融法在测定贫营养水体叶绿素 a 浓度上具有一定的优势 (采用反复冻融法建议贫营养水体过滤水量为 $500 \sim 1000 \mathrm{ml}^{[8]}$ ), 但当叶绿素 a 浓度较高时, 微波法提取率显 著高于反复冻融法, 测定结果的相对偏差较小, 且提取时间较冻融法缩短一半以上, 适用于富营养化水体的 应急监测.

\section{4 参考文献}

[ 1 ] SEPA "Aquatic Organisms Monitoring Handbook" editorial ed. Aquatic organisms monitoring handbook. Nanjing: Southeast University Press, 1993: 177-180(in Chinese). [ 国家环境保护局《水生生物监测手册》编委会. 水生生物监测手册. 南京: 东南大学出版社, 1993: 177-180.]

[ 2 ] Kim Sangchan, Tu Qingying eds. Eutrophication survey specifications (2nd edition). Beijing: China Standard Press, 1990: 268-270 (in Chinese). [ 金相灿, 屠清瑛. 湖泊富营养化调查规范(第 2 版). 北京: 中国标准出版社, 1990: 268-270.]

[ 3 ] Huang Yingbo. An improved method for extraction of chlorophyll a in phytoplankton: freezing-thawing method. China Sci- 
ence and Technology Information, 2012, (10): 157(in Chinese with English abstract). [ 黄莹波. 浮游植物叶绿素 a 提 取方法的改进: 反复冻融一浸提法. 中国科技信息, 2012, (10) : 157.]

[ 4 ] Rosen BH, Lowe RL. Physiological and ultrastructural responses of Cyclotella meneghiniana (Bacillariophyta) to light intensity and nutrient limitation. Journal of Phycology, 1984, 20(2) : 173-183.

[ 5 ] Levassseur M, Thompson PA, Harrison PJ. Physiological acclimation of marine phytoplankton to different nitrogen sources. Journal of Phycology, 1993, 29(5) : 587-595.

[ 6 ] Pápista É, Ács É, Böddi B. Chlorophyll-a determination with ethanol—a critical test. Hydrobiologia, 2002, 485 : 191-198.

[ 7 ] Feng Jing, Li Yanbo, Zhu Qing et al. Comparison of methods for phytoplankton chlophyll-a concentration measurement. Ecology and Environment, 2008, 17(2): 524-527(in Chinese with English abstract). [冯菁, 李艳波, 朱擎等. 浮游植物 叶绿素 a 测定方法比较. 生态环境, 2008, 17(2) : 524-527.]

[ 8 ] Lin Shaojun, He Lijing, Huang Peisheng et al. Comparison and improvement on the extraction method for chlorophyll $a$ in phytoplankton. Ecology and Environment, 2005, 24(1):9-11 (in Chinese with English abstract). [林少君, 贺立静, 黄 沛生等. 浮游植物中叶绿素 a 提取方法的比较与改进. 生态科学, 2005, 24(1) : 9-11.]

[ 9 ] Ganzler K, Salgó A, Valkó K. Microwave extraction A novel sample preparation method for chromatography. Journal of Chromatography A, 1986, 371: 299-306.

[10] Xie Mingyong, Chen Yi. The research progress of microwave assisted extracting technology. Journal of Food Science and Biotechnology, 2006, 25(1) : 105-114(in Chinese with English abstract). [谢明勇, 陈奕. 微波辅助萃取技术研究进展. 食品与生物技术学报, 2006, 25(1): 105-114.]

[11] Eskilsson CS, Björklund E. Analytical-scale microwave-assisted extraction. Journal of Chromatography A, 2000, 902 (1) : 227-250.

[12] Ren Qingguo. The study of microwave assistant extraction on chlorophyll from silkwarm and chlorophyll-copper-sodium preparation[Dissertation]. Dalian: Dalian University of Technology, 2005 (in Chinese with English abstract). [任清国. 微 波辅助萃取昙沙中叶绿素及叶绿酸铜钠的研究 [ 学位论文]. 大连: 大连理工大学, 2005.]

[13] Pasquet V , Chérouvrier JR, Farhat F et al. Study on the microalgal pigments extraction process: performance of microwave assisted extraction. Process Biochemistry, 2011, 46(1) : 59-67.

[14] Jiang Xiuxin ed. Microwave repair atlas. Harbin: Harbin Engineering University Press, 2006: 31(in Chinese). [蒋秀欣. 微波炉维修图集. 哈尔滨: 哈尔滨工程大学出版社, 2006: 31.]

[15] Li Zhenguo, Lu Jun, Wang Guoxiang et al. Comparison of measurement of phytoplankton chlorophyll-a concentration by spectrophotometry. Environmental Monitoring in China, 2006, 22(2) : 21-23(in Chinese with English abstract). [李振 国, 卢军, 王国祥等. 分光光度法测定浮游植物叶绿素 a 的比较研究. 中国环境监测, 2006, 22(2) : 21-23.]

[16] Zhang Wuchang, Wang Rong. Effect of light and temperature on the degradation of chlorophyll a and phaeophorbidea. Marine Science, 2000, 24(4) : 50-52 (in Chinese with English abstract). [张武昌, 王荣. 光和温度对叶绿素 a 和脱镁叶 绿素 a 降解的影响. 海洋科学, 2000, 24(4): 50-52.]

[17] Sun Liqin, Wang Changhai, Jiang Tao. Research on the methods of Porphyridium cruentum cells fragmentation. Marine Science Bulletin, 2004, 23(4) : 71-74(in Chinese with English abstract). [孙利芹, 王长海, 江涛. 紫球藻细胞破碎方法 研究. 海洋通报, 2004, 23(4): 71-74.] 